Research Article

Xiaojie Li*

\title{
Research on the emotional tendency of web texts based on long short-term memory network
}

https://doi.org/10.1515/jisys-2021-0088

received May 31, 2021; accepted July 24, 2021

\begin{abstract}
Through the analysis of emotional tendency in online public opinion, governments and enterprises can stabilize people's emotion more effectively and maintain social stability. The problem studied in this paper is how to analyze the emotional tendency of online public opinion efficiently, and finally, this paper chooses deep learning algorithm to perform fast analysis of emotional tendency of online public opinion. This paper briefly introduced the structure of the basic model used for emotional tendency analysis of online public opinion and the convolutional neural network (CNN) model used for text emotion classification. Then, the CNN model was improved by long short-term memory (LSTM). A simulation experiment was carried out on MATLAB for the improved text emotion classification model to verify the influence of activation function type on the improved model and the performance difference between the improved model and support vector machine (SVM) and traditional CNN models. The results showed that the improved classification model that adopted the sigmoid activation function had higher accuracy and was less affected by language than the relu and tanh activation functions; the improved classification model had the highest accuracy, recall rate, and $F$-value in classifying emotional tendency of web texts, followed by the traditional CNN model and the SVM model.
\end{abstract}

Keywords: online public opinion, emotional tendency, CNN, LSTM

\section{Introduction}

With the development of the Internet, people are acquiring information faster and faster, and the amount of information they acquire is also increasing. On the one hand, the rapid access to massive information has facilitated most people's lives, but on the other hand, it has become easier to spread untrue rumors [1]. Online public opinion refers to the tendentious and influential common opinions formed by the public on certain social events through the Internet. Due to the complexity of interest relationships, online public opinion is likely to cause dilemmas for individuals, enterprises, or governments [2]. In particular, online public opinion caused by false or one-sided untrue information can cause serious adverse consequences. Online public opinion is a kind of group opinion, which is composed of different individual opinions. Although different individual opinions have different emotional connotations, they converge to show emotional tendencies as a whole, reflecting most people's emotional feedback on the event [3]. Through the analysis of emotional tendencies in online public opinion, we can effectively understand the impact of public policies or events involving public interests on the masses, and thus take targeted measures. Xu et al. [4] proposed a term weighting method based on improved term frequency-inverse document frequency for

\footnotetext{
* Corresponding author: Xiaojie Li, Education and Teaching Department, Zhengzhou Preschool Education College, No. 9, Shimin Xincun North Street, Jinshui District, Zhengzhou, Henan 450000, China, e-mail: fpjx41781@sina.cn
} 
Tibetan text information extraction for extracting hot words in Tibetan public opinion. The experimental results showed that the method was effective in extracting Tibetan hot words and had a high accuracy in classifying Tibetan public opinion. Xia et al. [5] put forward a theme analysis model for lyrics and an emotional evolution model for lyrics, which are rich in information and prone to change over time. The results of the case analysis showed that the model effectively addressed the immaturity and inaccuracy of the current public opinion analysis. Rao et al. [6] proposed a topic-level maximum entropy (TME) model for socio-emotional classification of short texts. Experiments on real short documents verified the effectiveness of TME in the socio-emotional classification of sparse words. This paper briefly introduced the basic model structure used for emotion analysis of online public opinion and the convolutional neural network (CNN) model used for text emotion classification. The CNN model was improved by long short-term memory (LSTM). Finally, a simulation experiment was carried out on MATLAB for the improved text emotion classification model.

\section{Emotion computing model of web texts}

Although the emotional dictionary is simple and fast to use, the rapid development of the Internet has promoted a large number of new words, and the frequency of dictionary updates has greatly increased. The updating of the dictionary requires a lot of workforce; therefore, this method is difficult to adapt to today's online public opinion analysis [7]. The emergence of intelligent learning algorithms reduces the demand for human resources. Figure 1 shows the basic model used to calculate emotional tendency in online public opinion. The basic process is text data collection - data preprocessing - text vectorization (or feature extraction) - text classification algorithm - emotional tendency classification results.

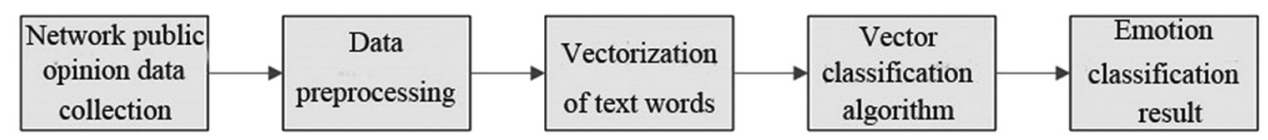

Figure 1: The basic model of emotional tendency calculation in online public opinion.

The first step is text data collection, which is usually implemented by crawler software. Then the text information collected by the crawler is preprocessed, including removing noise data and meaningless symbols, to prevent these invalid data from affecting the subsequent classification. After "cleaning" the text information, the short sentences and long sentences are segmented [8], and the divided words are vectorized (or feature vectors are extracted). The reason for word segmentation is that there is no space between words in Chinese sentences. Without lexical vectorization, the computer would rigidly treat the whole sentence as a word, affecting classification accuracy. The purpose of word vectorization is to transform the natural language of texts into machine language that computers can distinguish [9].

The traditional approach to word vectorization is the one-hot encoding method [10]. The principle of the one-hot encoding method is to use a sufficiently large vocabulary as the coordinate axis and representing words with the coordinates on the coordinate axis. For example, as shown in Table 1, it is assumed that there are six words in the constructed vocabulary, i.e., the vector used for representing a word is sixdimensional. The vector of "hello" is $(1,0,0,0,0,0)$, the vector of "ha-ha" is $(0,0,1,0,0,0)$, and so on.

Table 1: Vocabulary of six-dimensional vector

\begin{tabular}{lllllll}
\hline $\begin{array}{lllll}\text { Vector dimension number } \\
\text { Word }\end{array}$ & 1 & 2 & 3 & 4 & 5 & 6 \\
& Hello & Bye & Ha-ha & Agree & Take off & Fall \\
\hline
\end{tabular}


From the perspective of the principle, as long as the vocabulary is large enough, it can cover as many words as possible and turn the text into a set of high-dimensional vectors. However, the disadvantages of this method can also be seen from the principle. In order to accurately convert textual information into vectors, the constructed vocabulary needs to be large enough, which also means that the dimension of the vector will be large and sparse. In addition, the vector converted by this method only refers to a word in the vocabulary and does not reflect the meaning of the word and the artistic conception embodied in the statement environment.

The text vectors transformed by the traditional one-hot encoding method are not only multidimensional and sparse, but also the transformed word vectors are independent of each other and cannot reflect their own meanings and connections with the context. In order to preserve as much as possible the meaning of words and the connection with the context when converting text vectors, this paper used Word2Vec to vectorize the text [11]. The basic structure is shown in Figure 2. The central words are input in the input layer, the vector of words is calculated in the hidden layer, and the predicted contextual words are output in the output layer. The basic steps of vectorization of words with this model are as follows: (1) the words in the corpus are input as the central words in the input layer; (2) the vector of the central words is calculated in the hidden layer (the dimension of the vector depends on the demand; the more the dimension is, the more accurate the distribution of lexical-semantic features can be reflected by the vector, but the more difficult the calculation will be); (3) the words before and after the central word are output according to the calculation results of the hidden layer; (4) the contextual words obtained by calculation are compared with those in the corpus, and the vector of the central word in the hidden layer is adjusted according to the error; (5) the above steps are repeated until the error is eliminated. After training, the central word vector in the hidden layer is the desired word vector. In short, when Word2Vec transforms text into vectors, it is trained to adjust the low-dimensional real vectors possessed by the words to be converted iteratively in the hidden layer of the training model with the context as the target until the target is reached. The purpose of Word2Vec training is not to accurately predict the context using the central word [12], but to get the low-dimensional real number vector of central words in the hidden layer after connecting the central words with the context, i.e., the hidden layer of Word2Vec after training is the ultimate goal.

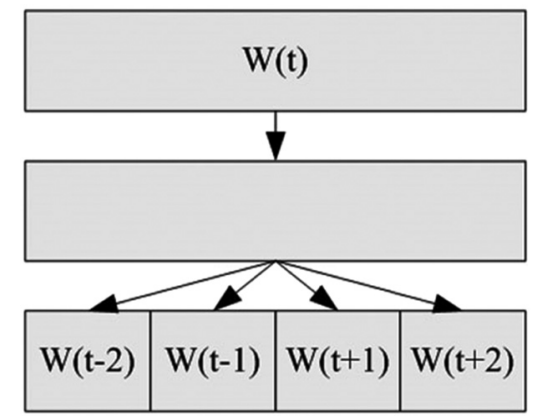

Input layer

Hidden layer

Output layer

Figure 2: The Skip-gram model in Word2Vec.

\section{Text emotion computing based on deep learning}

\subsection{Two-dimensionalization of text vector}

Deep learning generally refers to learning algorithms based on neural networks. Neural networks simulate the processing of information by neurons in the human brain and are well adapted for various nonlinear problems with hidden laws. In this paper, the CNN algorithm is selected to identify and classify the emotional tendencies of online public opinion [13]. The initial use of the CNN algorithm is for image recognition; therefore, before it is used in text recognition, simple processing of texts is needed, i.e., arrange 
it into a two-dimensional matrix [14]. Taking a four-dimensional text vector as an example, it is assumed that the text sentence to be detected can be divided into at most four words, then the two-dimensional vector matrix of the sentence is shown in Figure 3. $w_{i}$ refers to a word in the sentence and $\left[x_{i 1}, x_{i 2}, x_{i 3}, x_{i 4}\right]$ is the four-dimensional vector of the corresponding word. All the text vectors processed by Word2Vec can be converted to a two-dimensional matrix in a size of $M \times N(M$ is the maximum number of words in a text, and $N$ is the vector dimension of words in the training process). In the process of conversion, if the number of words in a text is less than $M$ after segmentation, then the remaining vectors in the matrix are denoted as 0 . The twodimensional matrix of text vectors is similar to an image, and each dimensional vector of each word is equivalent to a pixel in an image, so the CNN algorithm can process the text vectors just like an image.

$$
\begin{aligned}
& w_{1} \\
& w_{2} \\
& w_{3} \\
& w_{4}
\end{aligned}\left[\begin{array}{llll}
x_{11} & x_{12} & x_{13} & x_{14} \\
x_{21} & x_{22} & x_{23} & x_{24} \\
x_{31} & x_{32} & x_{33} & x_{34} \\
x_{41} & x_{42} & x_{34} & x_{44}
\end{array}\right]
$$

Figure 3: Text vectors arranged as a two-dimensional matrix.

\subsection{The basic process of the traditional CNN algorithm}

The two-dimensional matrix of the preprocessed text vector is input to the input layer of the CNN algorithm. Convolution computation is performed on the vector matrix using convolution kernels in the convolution layer to extract the text features. Then, the text feature graph is transferred to the pooling layer for down sampling operation [15]. The main function of the pooling layer is to compress the text features and reduce the computational effort. The convolution and down sampling operations are repeated several times according to the requirements, and the classification is performed in the fully connected layer using softmax. Finally, the results are output in the output layer. If it is in the process of training, the weight parameters in the CNN algorithm are adjusted according to the error between the output results and the standard results. After repeated training, the algorithm is used according to the above process.

\subsection{The LSTM-improved CNN algorithm}

In order to improve the classification performance of the CNN algorithm for text emotion tendency, this paper introduces LSTM [16]. The main function of LSTM is to highlight the key semantic features and reduce the interference of useless features, thus making full use of the semantics in the text vector and the contextual connections in the context.

The training process of the LSTM-improved CNN algorithm in classifying the emotion tendency of online public opinion is shown in Figure 4.

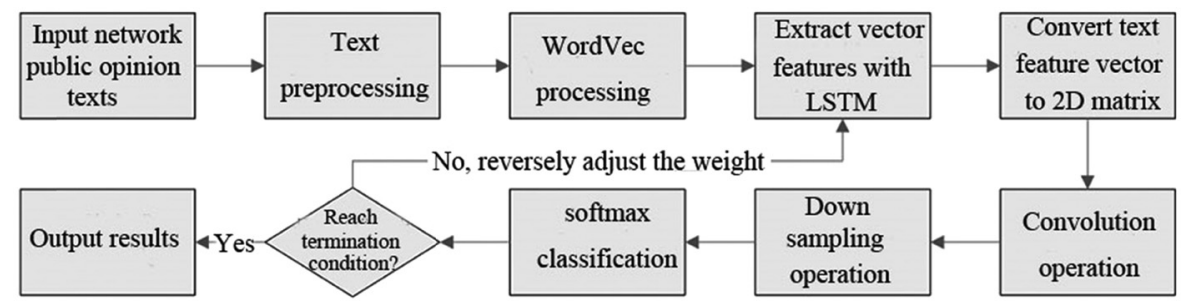

Figure 4: The training process of online public opinion emotion tendency classification model based on the LSTM-improved CNN algorithm. 
(1) The required text data are collected by crawler software.

(2) The data are preprocessed.

(3) The natural language is converted into vector language in machine language.

(4) The text vector is input into the LSTM layer of the improved classification model for feature extraction. LSTM includes input gate, forgetting gate, and output gate. The function of the input gate is to determine the number of new information added to cells, the function of the forgetting gate is to determine the number of original information discarded, and the function of the output gate is to output the final output after synthesizing the first two gates. The specific formula is:

$$
\left\{\begin{array}{l}
i_{t}=g\left(\omega_{i} \cdot\left[h_{t-1}, x_{t}\right]+b_{i}\right) \\
\tilde{C}_{t}=\tanh \left(\omega_{C} \cdot\left[h_{t-1}, x_{t}\right]+b_{C}\right) \\
C_{t}=f_{t} \cdot C_{t-1}+i_{t} \cdot \tilde{C}_{t} \\
f_{t}=g\left(\omega_{f} \cdot\left[h_{t-1}, x_{t}\right]+b_{f}\right) \\
o_{t}=g\left(\omega_{o} \cdot\left[h_{t-1}, x_{t}\right]+b_{o}\right) \\
h_{t}=o_{t} \cdot \tanh \left(C_{t}\right),
\end{array}\right.
$$

where $x_{t}$ is the current cell input, $h_{t-1}$ is the state of the previous hidden layer, $C_{t-1}$ is the previous cell state, $i_{t}$ is the proportion that determines the amount of the newly added information that can be kept in mind, $\tilde{C}_{t}$ is the cell state of the newly added information, $C_{t}$ is the cell state after adding new information, $\omega_{i}$ and $\omega_{t}$ are the corresponding weights, $b_{i}$ and $b_{C}$ are the corresponding offsets, $f_{t}$ is the proportion of information not to be forgotten; $\omega_{f}$ is the corresponding weight, $b_{f}$ is the corresponding offset, $o_{t}$ is the proportion that determines the amount of the final output information, and $h_{t}$ is the final output or the next hidden layer state.

(5) The text vector after LSTM feature extraction is transformed into a two-dimensional matrix and input into the convolution layer of the classification model for convolution operation:

$$
x_{j}^{l}=f\left(\sum_{j \in M} x_{i}^{l-1} \cdot W_{i j}^{l}+b_{j}^{l}\right),
$$

where $x_{j}^{l}$ is the feature graph obtained by convolution kernel convolution, $x_{i}^{l-1}$ is the feature output after the last convolution and pooling, $W_{i j}^{l}$ is the weight parameter, $b_{j}^{l}$ is the offset, $M$ is the number of convolution kernels of the l-th convolution layer, and $f(\bullet)$ is the activation function.

(6) In the pooling layer, features are processed by mean pooling to compress data.

(7) Steps (5) and (6) are repeated according to the number of convolution layer and pooling layer in the classification model. After the final pooling layer compresses the feature graph, it is input into the fully connected layer to classify text emotion by the softmax function, and the result is output to the output layer.

(8) Whether the training of the classification model can be finished is determined. If it can, the training results and classification model are output; otherwise, the parameters of the LSTM layer and convolution layer were adjusted to repeat steps (4)-(7). The parameters of the classification model are adjusted according to the error between the output result of the output layer and the expected result. As the model is used for classification, cross-entropy is used to calculate the error, and the parameters are adjusted reversely.

\section{Simulation experiment}

\subsection{Experimental environment}

The improved online public opinion emotion tendency classification model was simulated and analyzed by MATLAB software [17]. The experiment was carried out in a laboratory server, configured with Windows 7 system, i7 processor, and $16 \mathrm{G}$ memory. 


\subsection{Experimental data}

The web public opinion texts used in this experiment were obtained from the application programming interface (API) of Weibo using crawler software. The data set contained 15,000 Weibo data, each of which consists of a plural number of sentences. There were a total of 40,000 sentences in the data set. Then the data set was divided into a training set and a test set. There were 8,000 Weibo data in the training set and 7,000 Weibo data in the test set. The text data collected from Weibo were in Chinese. In the Internet communication environment that breaks national boundaries, the English text will inevitably appear in online public opinion. Analysis of English online public opinion is equally important; therefore, in order to test the effectiveness of the improved emotional tendency classification model on English texts, the reviews on foreign English films were also crawled using crawler software, and the emotion tags were classified. Some reviews that did not contain emotions such as anger, happiness, worry, calm, fear, and mourning were excluded. Finally, 15 thousand reviews were obtained, and they were divided into a training set including 8,000 reviews and a test set including 7,000 reviews.

\subsection{Experimental setup}

Table 2 shows the basic structural parameters of the text emotion tendency classification model. Word2Vec used in text preprocessing used 300-dimensional vectors to transform words. The convolution layer and pooling layer were set as three layers. Every convolution layer contained 32 convolution kernels in a size of $2 \times 2$. The down sampling operation of the pooling layer was mean pooling, the size of the pooling box was $2 \times 2$, and the moving step length was 2 . The size of the data in the two-dimensional matrix input into the input layer was $M \times 300$. In the LSTM layer, there were 64 hidden neurons, the weight was initialized using glotot_normal, and the offset was set as 0 .

Table 2: The basic structural parameters of the classification model

\begin{tabular}{llll}
\hline Structure name & Setup & Structure name & Setup \\
\hline Convolution layer & Three layers & Convolution kernel & 32 , a size of $2 \times 2$ \\
Pooling layer & Three layers; mean & Pooling box & A size of $2 \times 2$; moving step \\
& pooling & length: 2 \\
Vector dimension of word2vec & 300 & Size of data in the input layer & $\mathrm{M} \times 300$ \\
\hline
\end{tabular}

\subsection{Experimental projects}

\subsubsection{Influence of activation function type on the classification model}

In the CNN algorithm part of the improved classification model, sigmoid, relu, and tanh activation functions were used, respectively. The influence of the improved classification model on text emotion classification under different activation functions was compared.

\subsubsection{Performance comparison between the improved classification model and other classification models}

In order to further verify the effectiveness of the improved classification model for emotion classification, in addition to the previous comparison experiment, this paper also conducted comparison experiments with 
the SVM model and the traditional CNN model. The two models used for comparison preprocessed and vectorized texts in the same way as the improved classification model. The basic parameters of the traditional CNN model were consistent with the structural parameters of the improved CNN model, and the activation function was sigmoid. For the SVM model, the sigmoid kernel function was used, and the penalty parameter was set as 1 .

\subsection{Experimental results}

It was seen from Figure 5 that for the same test set, Chinese Weibo text or English film review text, the classification model that adopted the sigmoid activation function had higher accuracy of emotion classification. It was found that the improved classification model was more accurate in classifying English film reviews regardless of the activation function used.

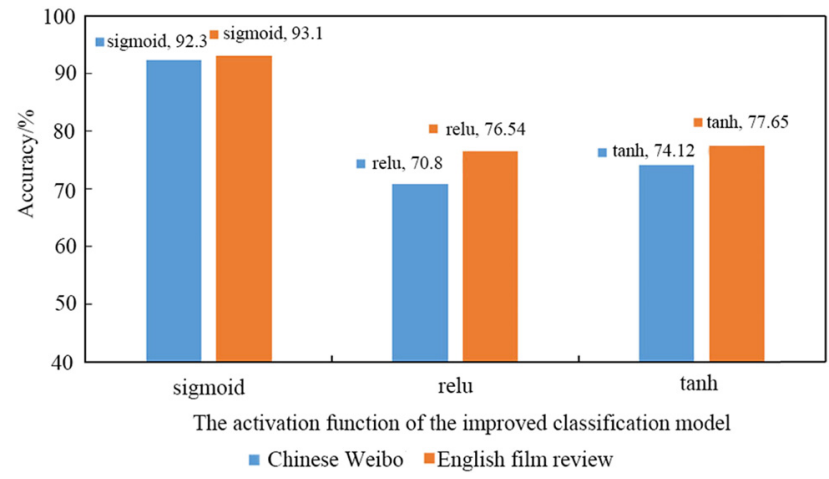

Figure 5: The influence of different activation functions on the accuracy of the improved classification model.

Limited by the space, this paper only showed the emotion classification results of the three classification models for Chinese and English texts in the test set, as shown in Table 3. It was seen from Table 3 that the improved CNN model accurately classified all the texts in Table 3, while the other two classification models had more or less errors in judgment.

Table 3: Partial emotion classification results of Chinese and English texts by three classification models

\begin{tabular}{|c|c|c|c|c|c|}
\hline Content & & $\begin{array}{l}\text { Standard } \\
\text { judgment }\end{array}$ & SVM model & $\begin{array}{l}\text { Traditional CNN } \\
\text { model }\end{array}$ & $\begin{array}{l}\text { Improved CNN } \\
\text { model }\end{array}$ \\
\hline \multirow[t]{3}{*}{ Chinese } & $\begin{array}{l}\text { This is not only corruption, but also the } \\
\text { destruction of the existing system! }\end{array}$ & Anger & Worry & Anger & Anger \\
\hline & Happy life, treat yourself well & Happiness & Happiness & Happiness & Happiness \\
\hline & $\begin{array}{l}\text { The patient on bed No. } 7 \text { was too late to } \\
\text { be rescued and left }\end{array}$ & Mourning & Calm & Fear & Mourning \\
\hline \multirow[t]{3}{*}{ English } & This movie is wonderful & Happiness & Happiness & Happiness & Happiness \\
\hline & The plot is terrible & Fear & Worry & Fear & Fear \\
\hline & This character, this is hateful & Anger & Fear & Calm & Anger \\
\hline
\end{tabular}

Table 2 illustrates the emotion tendency classification results of the three models, and Figures 6-8 show the accuracy, recall rate, and $F$-value of the three models in classifying text emotion tendency. It was seen from Figure 6 that the classification accuracy of the improved CNN model was the highest, followed by 


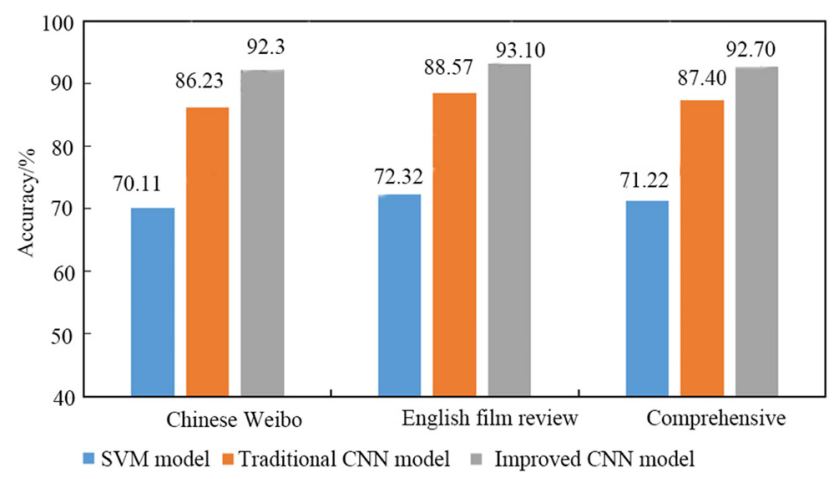

Figure 6: The accuracy of three classification models for text emotional tendency classification.

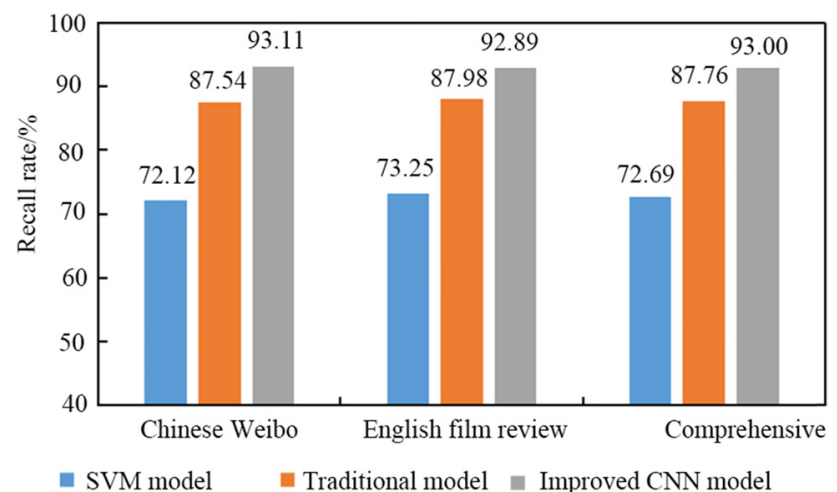

Figure 7: The recall rate of three classification models for text emotional tendency classification.

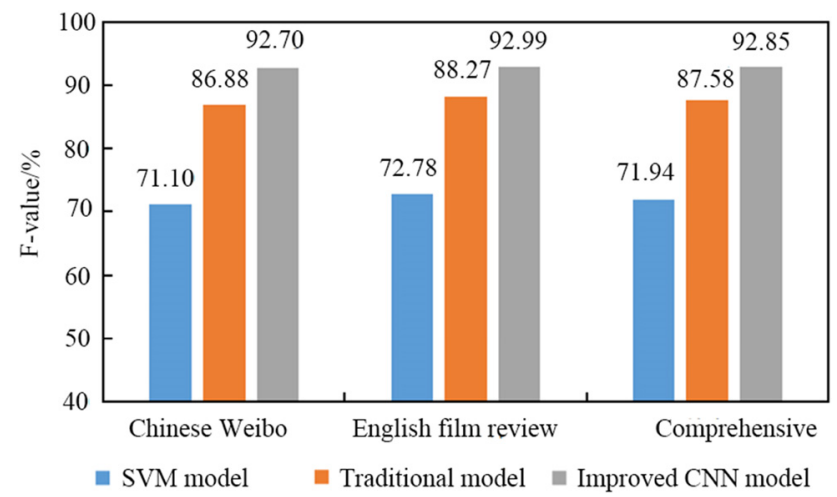

Figure 8: The $F$-value of three classification models for text emotional tendency classification.

the traditional CNN model and the SVM model; the sorting of the recall rate and $F$-value was the same. By comparing the classification accuracy of Chinese and English texts under the same classification model in Figures 6-8, it was found that the accuracy, recall rate, and $F$-value of the three classification models were higher in analyzing the emotion of English film reviews, but the difference was not much. 


\section{Discussion}

The advent of the Internet has greatly facilitated communication and has lowered the threshold for people to express their opinions on public events, allowing more people to express their opinions freely [18]. While the number of opinions that can be collected has increased significantly by lowering the threshold for expressing opinions, the number of worthless opinions, or opinions with obvious inflammatory sentiments, has also increased at the same time. In addition, the lowering of the threshold for expressing opinions also means that some mentally immature teenagers are able to watch worthless or even emotionally inflammatory opinions. Once these messages with negative emotions or even rumors spread, it will inevitably cause damage to the network environment, and if left unchecked, it may also spread to reality. Therefore, the analysis of emotional tendencies contained in public opinion in the Internet can help in the management of the online environment. However, the huge amount of data generated per unit time in the Internet makes it impossible to analyze online public opinion through manual means [19]. In order to make fast and accurate analysis of emotional tendencies in online public opinion, this paper adopted an intelligent algorithm to identify online public opinion. In this paper, we first proposed to vectorize the opinion text in two dimensions and then used the CNN algorithm to identify the emotional tendency of the text. Meanwhile, this paper improved the traditional CNN algorithm, by introducing the LSTM algorithm for feature extraction of text vectors and then converting the features into a two-dimensional matrix.

In order to examine the performance of the improved CNN algorithm for analyzing public opinion, simulation experiments were conducted, and the improved CNN algorithm was also compared with SVM and traditional CNN algorithms. The final experimental results have been shown above. The comparison of the activation functions used in the improved CNN algorithm showed that the highest accuracy was achieved for Chinese and English texts when the sigmoid function was used as the activation function. The reason for the above result was that English words were separated from each other by spaces, which could be vectorized without special word segmentation, but Chinese words were closely related to each other and needed word segmentation. Different word segmentation could affect the meaning of words in sentences and increase the difficulty of emotion classification. However, it was also seen from the figure that the improved classification model that adopted the sigmoid activation function did not have as obvious a classification accuracy gap between Chinese and English texts as the models that adopted the other two activation functions, i.e., the sigmoid function was relatively less affected by text language, which was more suitable for the improved online public opinion emotion tendency classification model.

The comparison of the improved CNN algorithm with SVM and traditional CNN algorithms showed that the improved CNN algorithm had the highest accuracy, recall rate, and $F$-value when facing Chinese and English texts. The reason for the higher accuracy was that the English text did not need special word segmentation, and the meaning of Chinese words would be affected by word segmentation. The reason for the small difference was that all the three models used the sigmoid function that could effectively map the text vector to a high-dimensional space, and it was closer to the nonlinear law after mapping.

In this paper, the intelligent algorithms used for online opinion analysis in Internet networks were studied, and the traditional CNN algorithm was improved using LSTM. The results of the final simulation experiments also verified the effectiveness of the improved CNN algorithm for the recognition of emotional tendencies in online opinion. This work provides an effective reference for improving the efficiency of online opinion control.

\section{Conclusion}

This paper briefly introduced the structure of the basic model for emotional tendency analysis of online public opinion and the CNN model used for text emotion classification, improved the CNN model with LSTM, and carried out a simulation experiment on the improved text emotion classification model in MATLAB to verify the influence of activation function type on the improved model and the performance 
difference between the improved model and SVM and traditional CNN models. The results showed that: (1) the classification model using the sigmoid activation function had the highest classification accuracy for text emotions, and the difference between the classification accuracy of Chinese and English texts was relatively small; (2) the improved CNN model had the highest classification accuracy, recall rate, and $F$-value for text emotional tendencies, followed by the traditional CNN model and the SVM model; (3) for online texts in different languages, the three classification models had slightly higher accuracy, recall rate, and $F$-value for English text emotion classification, but the difference was not large.

Conflict of interest: The author state no conflict of interest.

\section{References}

[1] Charoensuk J, Sornil O. A hierarchical emotion classification technique for Thai reviews. J ICT Res Appl. 2018;12(3):280.

[2] Xu R, Chen T, Xia Y, Lu Q, Liu B, Wang X. Word embedding composition for data imbalances in sentiment and emotion classification. Cogn Comput. 2015;7(2):226-40.

[3] Li X, Rao Y, Xie H, Liu X, Wong TL, Wang FL. Social emotion classification based on noise-aware training. IoT. 2019;123:100121.

[4] Xu G, Wang C, Yao H, Qi Q. Research on Tibetan hot words, sensitive words tracking and public opinion classification. Cluster Comput. 2017;22(3):9977-90.

[5] Xia X, Gu X, Lu Q. Research on the model of lyric emotion algorithm. J Phys Conf Ser. 2019;1213(4):042004.

[6] Rao Y, Xie H, Li J, Jin FM, Wang FL, Li Q. Social emotion classification of short text via topic-level maximum entropy model. Inform Manage. 2016;53(8):978-86.

[7] Lai Y, Zhang L, Han D, Zhou R, Wang G. Fine-grained emotion classification of Chinese microblogs based on graph convolution networks. World Wide Web. 2020;23(4):2771-87.

[8] Wei DZ, Chen FJ, Zheng XX. Internet public opinion chaotic prediction based on chaos theory and the improved radial basis function in neural networks. Acta Phys Sin. 2015;64(11):1-8.

[9] Jang K, Park S, Kim WJ. Automatic construction of a negative/positive corpus and emotional classification using the internet emotional sign. J KIISE. 2015;42(4):512-21.

[10] He H. Research on prediction of internet public opinion based on grey system theory and fuzzy neural network. J Intell Fuzzy Syst. 2018;35(1):1-8.

[11] Gao X, Fu L. Methods of uncertain partial differential equation with application to internet public opinion problem. J Intell Fuzzy Syst. 2017;33(3):1-11.

[12] Lian Y, Dong X, Liu Y. Topological evolution of the internet public opinion. Physica A. 2017;486:567-78.

[13] Wang A, Liu X, Sun X, Wang J. Research of internet public opinion based on hybrid algorithm of LDA and VSM. Ce Ca. 2017;42(4):1508-13.

[14] Zhihui P. The approaches of internet public opinion research. Libraly J. 2016;35(12):63-8.

[15] Zhang F, Li SC, Guan Y. Hot issues about drug price in china: from the view of internet public opinion monitoring. Value Health. 2016;19(3):A273-4.

[16] Zhu H, Liu P, Shan X. Analysis of internet-based public opinion in China, 2012. J Mol Neurosci. 2015;49(3):614-7.

[17] Li L. Research on the transfer rules of internet users' negative emotional state in financial public opinion. Open J Bus Manag. 2020;8(1):282-301.

[18] Li J, Tang H, Tan H. Research on the evolution and prediction of Internet public opinion of major pandemics-taking the COVID-19 pandemic as an example. J Phys Conf Ser. 2021;1774(1):012038.

[19] Jia F, Chen CC. Emotional characteristics and time series analysis of internet public opinion participants based on emotional feature words. Int J Adv Robot Syst. 2020;17(1):72988142090421. 